Check for updates

New York City

Twitter @ekutscher

Cite this as: BMJ 2021;375:n3021 http://dx.doi.org/10.1136/bmj.n3021 Published: 06 December 2021

\title{
Preparing for Omicron as a covid veteran
}

\section{Eric Kutscher internal medicine physician}

It feels like the world is slowly closing in on me as I hear the ambulances pass my window at home. I remember the last time I felt this way-March 2020. News, social media, and texts from friends and work are all focused on the same thing: potential impending doom from covid-19. Except it's no longer the first, or second, or third wave. At this point, I've lost count. Omicron is coming, and I don't know if I'm ready.

During the first wave in New York, covid-19 was the "novel" coronavirus and I was a "healthcare hero," sent off to fight for the lives of my patients. We had no data, no knowledge, and no experience. Covid had the upper hand and claimed the lives of tens of thousands of people in my city. Yet I would go to work each day with a mix of fear and pride: my calling as a doctor is to help when others are in need, and I was literally putting my life at risk to do so. Without medications, vaccinations, or treatments, my physical presence was the most lifesaving gift I could provide.

Flash forward almost two years to now. Covid is no longer novel. I am no longer a healthcare hero, but instead an exhausted, weary doctor. We have medications, vaccinations, and treatments for covid. Yet, here we are, here I am, gearing up for another wave.

I miss the naïveté of my former self. Going to work was easier when I thought the pandemic was limited to one moment in time, something that would resolve. Treating patients with the same medical problem felt like more of an intellectual challenge then, as the intricate interplay between the virus and the human body unfolded in front of us. I believed that "science" and "evidence" could help guide us through the pandemic, and that I was part of the workforce helping us to understand more about covid-19.

But now I'm disillusioned. I've seen how our medical trends with how to treat covid-19 can change by the hour, with self-declared experts always ready to criticise decisions and cherry pick evidence to follow. I've watched how systematically hospital systems continue to prioritise efficiency, rankings, and profits over patient centred care. I've learnt that I am nothing but one of millions of healthcare workers expected to come to work every day based solely on my own goodwill. The calls for the protection of healthcare workers with life insurance, disability insurance, and student debt forgiveness have been forgotten just as quickly as they were proposed. Lacking these investments in my personhood, it's hard to feel like more than another faceless number in the system.

My patients are now jaded too, as politics has entered their hospital bed. Some ask for ivermectin and refuse to have conversations about quarantine for family members they have exposed to the virus. They "other" me, seeing me as part of the healthcare system that mocks the political right, rather than as another human being at their bedside feeling just as vulnerable as they are to the pandemic. My vaccinated patients remind me of their status over and over again, as if trying to clue me in to give them preferential treatment or empathy for having a breakthrough infection. I empathise with both my patients who are vaccinated and unvaccinated, but I still leave each room feeling a sense of defeat, powerlessness, and anger that the pandemic persists.

I remember the ambulances going by my window seemingly every minute in March 2020. I think of the death certificates I've signed of names I can't remember, the phone calls I made sharing life changing news to faces I've never seen. I still feel the chest compressions I've performed on bodies I've never known alive and the pulses I've only noticed once absent. These memories are ingrained in my personhood, now a part of who I am. I bring them with me to each new covid case. I add to them with each new covid wave.

I am a covid veteran. Watching the United States prepare for the new Omicron variant simultaneously sparks traumatic memories of the past and dread for the future. I see mistakes in our public health policies that mirror those from before yet no longer feel empowered to criticise or offer alternatives. The playbook developed in 2020 seems to be the only one we now know. History repeats itself because the irrational approaches taken previously are depicted as rational in hindsight.

As I gear up for Omicron, my body is bruised and my mind is distracted. I am not the same person I was during March 2020. The extra pounds and memories weigh on me. Yet I'm asked to do more this time-show up, knowing what the future looks like. Knowing that many of my patients may die. Knowing that caring for them will take a toll on my physical and mental health, and that I'll have to find ways to heal myself. I'm asked to show up to help with this new variant knowing that it's likely far from the last.

So, I put on my mask and gown and walk into my patient's room. Neither of us ever thought we'd be here, but we are. And for now, that is the common ground I can find peace in.

Competing interests: none declared. 